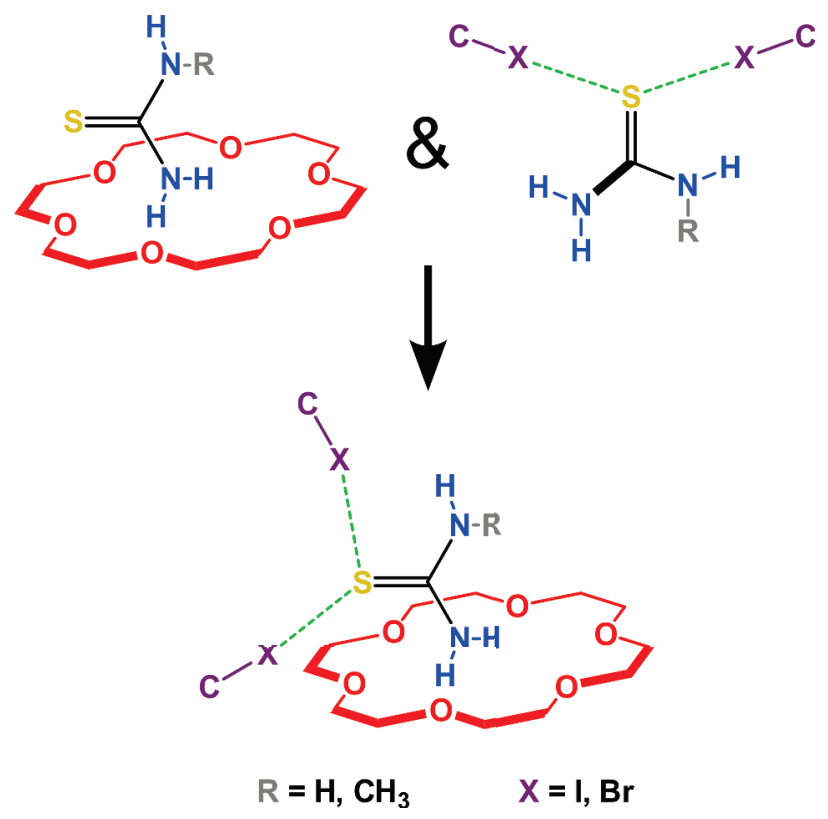

Figure 1. Hydrogen and halogen bonded motifs are combined in the studied ternary cocrystals.

Keywords: crystal engineering, ternary co-crystals, hydrogen bond, halogen bond, orthogonality

\section{MS32-O5 Halogen bonding in host-guest compounds: Structures and kinetics of enclathration and desolvation}

Francoise Mystere Amombo Noa ${ }^{1}$, Susan Ann Bourne ${ }^{1}$, Luigi Renzo Nassimbeni ${ }^{1}$

\section{Centre for Supramolecular Chemistry, Department of Chemistry, University of Cape Town, Rondebosch 7701, South Africa. \\ email: noa.mystere@gmail.com}

The host compounds tetrakis-(4-bromophenyl) ethylene and its iodo-analogue form inclusion compounds with a series of chloro- and iodo-methanes. Their structures have been elucidated and their non-bonded halogen $\cdots$ halogen contacts analysed and classified. Their kinetics of desolvation have been studied and the concomitant activation energies established. The velocity of the enclathration for the solid-methyl iodide vapour reactions and associate rate law have been established. Figure 1 shows halogen...halogen interactions between the host compound tetrakis-(4-bromophenyl) ethylene and the guest diiodomethane.

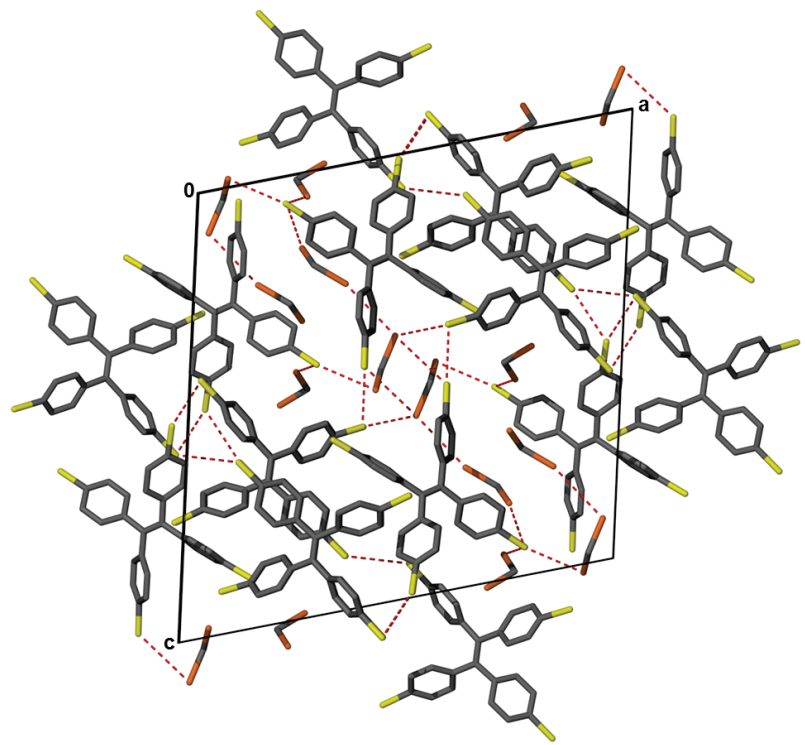

Figure 1. Packing diagram of tris diiodomethane bis tetrakis-(4-bromophenyl) ethylene along [010] showing halogen $\cdots$ halogen interactions.

Keywords: Halogen-halogen interactions, halogen bonding 